\title{
Acceleration of near-field scattering from an inhomogeneous spherical shell
}

\author{
M D SHARMA \\ Department of Mathematics, Kurukshetra University, India 136119. \\ e-mail: mohan_here@rediffmail.com
}

The three dimensional scattering of near-field, from a point source, is studied for acceleration in the time domain. The perturbation method is applied to define the acceleration for the first order scattering from a weak inhomogeneity in a homogeneous surrounding. A body force, arising from the interaction between the primary waves and the inhomogeneity, acts as the source generating the scattered motion. The acceleration of scattered waves is related to the velocity and density fluctuations of the inhomogeneity. No restrictions are placed on the inhomogeneity size or locations of the source and receiver. Decoupling of scattered motion enables the identification of different phases. Integral expressions are derived for the scattering acceleration due to the incidence of nearfield wave (from an impulsive point force) at a radially inhomogeneous volume element. These integrals are solved further for scattering from an inhomogeneous spherical shell. The accelerations for back scattering are obtained as a special case. These accelerations are simple analytically solvable expressions in closed form.

Only spherical asymmetry of $P$ wave velocity inhomogeneity can affect the scattered $S$ acceleration. Scattered $P$ acceleration is affected by the gradient of $S$ wave velocity inhomogeneity. The back scattering of near-field from a spherical shell, is independent of radial inhomogeneity of $P$ wave velocity. Inhomogeneity with smoothly perturbed $S$ wave velocity does not back-scatter any acceleration. Accelerations are computed numerically for scattering from a part of inhomogeneous spherical shell. Hypothetical models are considered to study the effects of the distances of spherical shell from source, receiver, its thickness and its position relative to the direction of impulsive force.

\section{Introduction}

The factors affecting any recorded motion are source effects, wave path effects and site response. The complex effects of wave paths do not affect the near-field studies. Hence, the data from the near-field offer better information on the parameters of the source in a nearby fault zone. Reservoir characterisation and non-destructive testing of materials are important examples of near-field studies. Elastic wave scattering could be an effective tool to characterise a material or a structure for any existing fault. The study of the dynamism at such a fault may help in improving the monitoring techniques used presently. This requires an advancement of scattering theory by incorporating the various aspects of physical property distributions in the theoretical models.

The modeling of waves scattered by a small inhomogeneity has taken a variety of approaches. Some of the approaches leading to advanced scattering theories are mentioned in an earlier paper of the author (Sharma 2004). The studies available in literature (Sato 1984; Wu and Aki 1985a, b, 1988; $\mathrm{Wu} 1989$ ) assume the point source to be either a $P$ wave source or $S$ wave source and, hence, discuss only the far-field scattering. Moreover, most of the analytical studies in scattering are in frequency domain whereas the experimental data are collected in time domain. This is a mismatch of the

Keywords. Scattering; near-field; acceleration; inhomogeneity.

J. Earth Syst. Sci. 114, No. 4, August 2005, pp. 401-410

(C) Printed in India. 
theory with the experiments. The time domain scenario always presents a more transparent picture of any process.

Sharma (2004) is, perhaps, the first such study that discussed the scattering of the near-field of a point source. In the paper, the author stressed the importance of near-field in scattering. The scattering of near-field of a point source from the spherical shell shaped inhomogeneity was studied to calculate the strains of scattered waves in time domain. In the work presented here, a similar study is considered, but to calculate the accelerations of scattered waves.

The author, here, derives the integral expression for acceleration of scattering, and solves it for the near-field term of the Green's function (Aki and Richards 1980) from an impulsive point source. Integral solutions are solved, further, for the scattering from an inhomogeneous spherical shell. The work presented is important on the following counts:

- Scattering is studied in time domain.

- Accelerations of different scattered phases are computed without any restriction on the position of receiver. Whereas, the studies available in literature compute displacement assuming both source and receiver far from the scatterer. Recording of acceleration as strong ground motion is necessary to solve the problems of seismic design and construction of safe structures.

- The near-field intermediate wave from point source is considered as a primary wave. When the source is very near to the inhomogeneity, this represents the ever ignored part of the scattering studies. Otherwise, this study is a complementary supplement to the far-field scattering approximations.

\section{First order scattering theory}

A finite volume weak inhomogeneity is considered in an isotropic elastic homogeneous medium of Lame's moduli $\lambda_{o}, \mu_{o}$ and density $\rho_{o}$. Elastic constants and density of this inhomogeneity are defined as

$$
p=p_{o}+\delta p,
$$

where $|\delta p|\left(\ll\left|p_{o}\right| ; p=(\lambda, \mu, \rho)\right)$, denote perturbations in elastic parameters and density. The inhomogeneity is weak so that scattered waves obey the wave equation of the unperturbed medium.

The displacements in the inhomogeneous medium are written as $\mathbf{u}=\mathbf{u}^{o}+\delta \mathbf{u}$, such that $|\delta \mathbf{u}| \ll\left|\mathbf{u}^{o}\right|$. The equation of motion in the inhomogeneous medium is given by

$$
\rho \ddot{u}_{i}=\rho f_{i}+\left(\lambda u_{j, j}\right)_{, i}+\left\{\mu\left(u_{i, j}+u_{j, i}\right)\right\}_{, j},
$$

where, $\mathbf{f}$ is the body force (per unit mass) representing the source fixed in the homogeneous part of the medium and generating primary waves.

For a weak inhomogeneity (ignoring terms of second and higher orders of perturbations), using (1), the equation (2) is resolved into two equations. These are

$$
\rho_{o} \ddot{\mathbf{u}}^{o}=\rho_{o} \mathbf{f}+\left(\lambda_{o}+\mu_{o}\right) \nabla\left(\nabla \cdot \mathbf{u}^{o}\right)+\mu_{o} \nabla^{2} \mathbf{u}^{o},
$$

and

$$
\ddot{\delta} \mathbf{u}=\left(\frac{\lambda_{o}+\mu_{o}}{\rho_{o}}\right) \nabla(\nabla \cdot \delta \mathbf{u})+\left(\frac{\mu_{o}}{\rho_{o}}\right) \nabla^{2} \delta \mathbf{u}+\mathbf{Q},
$$

where,

$$
\begin{aligned}
Q_{i}= & \left(\frac{\delta \rho}{\rho_{o}}\right)\left(f_{i}-\ddot{u}_{i}^{o}\right)+\left(\frac{\delta \lambda+\delta \mu}{\rho_{o}}\right) u_{j, i j}^{o} \\
& +\left(\frac{\delta \mu}{\rho_{o}}\right) u_{i, j j}^{o}+\left(\frac{\delta \lambda_{, i}}{\rho_{o}}\right) u_{j, j}^{o} \\
& +\left(\frac{\delta \mu_{, j}}{\rho_{o}}\right)\left(u_{i, j}^{o}+u_{j, i}^{o}\right),
\end{aligned}
$$

represents the body force (per unit mass) which is determined by the interaction of inhomogeneity with the primary wavelets from source.

Using (3) in (5), changing the elastic parameter perturbations to velocity perturbations and ignoring the terms of second and higher orders of perturbations, the scattering source is given by

$$
\begin{aligned}
Q_{i}= & \left(2 \alpha_{o} \delta \alpha\right) u_{j, i j}^{o}+\left(2 \alpha_{o} \delta \alpha+\alpha_{o}^{2} \frac{\delta \rho}{\rho_{o}}\right)_{, i} u_{j, j}^{o} \\
& +\left(2 \beta_{o} \delta \beta\right)\left(u_{i, j j}^{o}-u_{j, i j}^{o}\right) \\
& +\left(2 \beta_{o} \delta \beta+\beta_{o}^{2} \frac{\delta \rho}{\rho_{o}}\right)_{, j}\left(u_{i, j}^{o}+u_{j, i}^{o}-2 \delta_{i j} u_{k, k}^{o}\right) .
\end{aligned}
$$

From (4), we can write

$$
\begin{gathered}
\frac{\partial^{2}(\nabla \cdot \delta \mathbf{u})}{\partial t^{2}}=\alpha_{o}^{2} \nabla^{2}(\nabla \cdot \delta \mathbf{u})+\nabla \cdot \mathbf{Q}, \\
\frac{\partial^{2}(\nabla \wedge \delta \mathbf{u})}{\partial t^{2}}=\beta_{o}^{2} \nabla^{2}(\nabla \wedge \delta \mathbf{u})+\nabla \wedge \mathbf{Q},
\end{gathered}
$$

and equation (4) itself can be written as

$$
\ddot{\delta} \mathbf{u}=\alpha_{o}^{2} \nabla(\nabla \cdot \delta \mathbf{u})-\beta_{o}^{2} \nabla \wedge \nabla \wedge \delta \mathbf{u}+\mathbf{Q} .
$$


Applying grad to (7) and curl to (8), we obtain

$$
\frac{\partial^{2}[\nabla(\nabla \cdot \delta \mathbf{u})]}{\partial t^{2}}=\alpha_{o}^{2} \nabla^{2}[\nabla(\nabla \cdot \delta \mathbf{u})]+\nabla(\nabla \cdot \mathbf{Q})
$$

$$
\begin{aligned}
\frac{\partial^{2}[\nabla \wedge(\nabla \wedge \delta \mathbf{u})]}{\partial t^{2}}= & \beta_{o}^{2} \nabla^{2}[\nabla \wedge(\nabla \wedge \delta \mathbf{u})] \\
& +\nabla \wedge(\nabla \wedge \mathbf{Q}) .
\end{aligned}
$$

Solutions of (10) and (11), for the waves scattered from a small volume $V$ and recorded at $\mathbf{r}$, can be written as

$$
\begin{aligned}
\nabla(\nabla \cdot \delta \mathbf{u}(\mathbf{r}, t))= & \frac{1}{4 \pi \alpha_{o}^{2}} \int \frac{1}{|\mathbf{r}-\mathbf{q}|} \nabla(\nabla \cdot \mathbf{Q}) \\
& \left(\mathbf{q}, t-\frac{|\mathbf{r}-\mathbf{q}|}{\alpha_{o}}\right) d V(\mathbf{q}),
\end{aligned}
$$

and

$$
\begin{gathered}
\nabla \wedge(\nabla \wedge \delta \mathbf{u}(\mathbf{r}, t))=\frac{1}{4 \pi \beta_{o}^{2}} \int \frac{1}{|\mathbf{r}-\mathbf{q}|} \nabla \wedge(\nabla \wedge \mathbf{Q}) \\
\left(\mathbf{q}, t-\frac{|\mathbf{r}-\mathbf{q}|}{\beta_{o}}\right) d V(\mathbf{q}) .
\end{gathered}
$$

$V$ is the inhomogeneous region where $\mathbf{Q}$ is nonzero.

Using (12) and (13) in (9), the acceleration of scattering reaching at $\mathbf{r}$ is given by

$$
\begin{aligned}
\ddot{\delta} \mathbf{u}(\mathbf{r}, t)= & \frac{1}{4 \pi} \int \frac{1}{|\mathbf{r}-\mathbf{q}|} \nabla \\
& \left\{\nabla \cdot \mathbf{Q}\left(\mathbf{q}, t-\frac{|\mathbf{r}-\mathbf{q}|}{\alpha_{o}}\right)\right\} d V(\mathbf{q}) \\
& -\frac{1}{4 \pi} \int \frac{1}{|\mathbf{r}-\mathbf{q}|} \nabla \\
& \wedge\left\{\nabla \wedge \mathbf{Q}\left(\mathbf{q}, t-\frac{|\mathbf{r}-\mathbf{q}|}{\beta_{o}}\right)\right\} d V(\mathbf{q}) \\
& +\mathbf{Q}(\mathbf{r}, t) .
\end{aligned}
$$

It is an integral expression for acceleration of scattering in which various phases can be identified. The first integral represents the scattering received as compressional waves and the second integral represents the shear waves in scattering. The last term, i.e., $\mathbf{Q}$ is zero outside $V$.

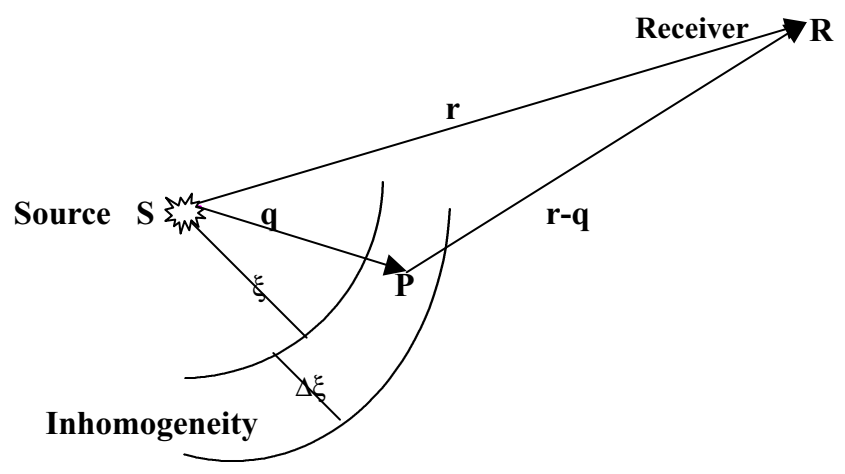

Figure 1. Geometry of the medium.

\section{Geometry of the medium (figure 1)}

Consider a point source at $S \leftrightarrow\left(S_{x}, S_{y}, S_{z}\right)$ in a Cartesian co-ordinate system $(x, y, z)$. The unit vector $\mathbf{n}=\left(n_{x}, n_{y}, n_{z}\right)$ defines the direction of the force at $S$. An arbitrary location for receiver is represented by $R \leftrightarrow\left(R_{x}, R_{y}, R_{z}\right)$. Hence, we have $\mathbf{r}=\left(R_{x}-S_{x}, R_{y}-S_{y}, R_{z}-S_{z}\right)$. An arbitrary point $P \leftrightarrow\left(P_{x}, P_{y}, P_{z}\right)$ in the inhomogeneity, is represented by the variable $\mathbf{q}=\left(P_{x}-S_{x}, P_{y}-S_{y}\right.$, $P_{z}-S_{z}$ ). In a local spherical co-ordinate system centered at $S$, the variable $\mathbf{q}=(q, \theta, \phi)$ is related to its Cartesian components by $q_{x}=q \sqrt{1-\chi^{2}} \cos \phi$, $q_{y}=q \sqrt{1-\chi^{2}} \sin \phi$ and $q_{z}=q \chi$. For a spherical shell inhomogeneity of thickness $\Delta \xi$ at radial distance $\xi$ from source, $q$ will be varying from $\xi$ to $\xi+\Delta \xi \cdot d V=q^{2} d q d \Omega$, where, $d \Omega=d \chi d \phi$ represents the solid angle subtended by the inhomogeneity at the source. For a complete spherical shell, $\chi(=\cos \theta)$ varies from -1 to 1 and $\phi$ varies from 0 to $2 \pi$.

\section{Acceleration of scattered near-field}

Following Sharma (2004), the displacement at a point $\mathbf{q}$ in the near-field of an impulsive point unit force at $S$ along the direction $\hat{\mathbf{n}}$, is given by

$$
\begin{aligned}
& \mathbf{u}^{o}(\mathbf{q}, t) \\
& \quad=4 \pi u_{o} \mathbf{d} \frac{s_{o}^{2} \alpha_{o}}{q^{3}} t\left[H\left(t-\frac{q}{\alpha_{o}}\right)-H\left(t-\frac{q}{\beta_{o}}\right)\right] .
\end{aligned}
$$

The meanings of variables and parameters involved are unchanged in the present study. Similarly, the scattering source is the body force $\mathbf{Q}$ generated by the interaction of primary wave, given by (15), with the inhomogeneity. The perturbation functions $F(q), G(q)$ and $E(q)$ are assumed to be thrice differentiable. The scattered waves from impulsive 
sources have their acceleration defined by the equation (4). The integral expression (14), calculates the scattering acceleration to be recorded at a receiver at $R$. Solving this equation, the scattering acceleration $\ddot{\delta} \mathbf{u}$ of $P$ and $S$ phases are obtained as follows:

$$
\begin{array}{r}
\delta \ddot{u}_{i}^{(j)}=u_{o} \alpha_{o} s_{o}^{2} \int \frac{X(q)}{q^{3}}\left[\chi_{f} \frac{q_{i}}{q} V_{j}+n_{i} W_{j}\right] d V(\mathbf{q}) ; \\
(j=P, S)
\end{array}
$$

where, $\chi_{f}=\hat{\mathbf{q}} \cdot \hat{\mathbf{n}}$ and $X(q)=1 /|\mathbf{r}-\mathbf{q}| \cdot V_{j}$ and $W_{j}$ are given by

$$
\begin{aligned}
V_{P}= & 4 \alpha_{o}^{2}\left[F Y_{q q q q}+\left(3 F^{\prime}-\frac{8}{q} F\right) Y_{q q q}\right. \\
& +\left(3 F^{\prime \prime}-\frac{16}{q} F^{\prime}+\frac{24}{q^{2}} F\right) Y_{q q} \\
& \left.+\left(F^{\prime \prime \prime}-\frac{8}{q} F^{\prime \prime}+\frac{24}{q^{2}} F^{\prime}-\frac{24}{q^{3}} F\right) Y_{q}\right] \\
& -24 \beta_{o}^{2}\left[\left(\frac{1}{q} G^{\prime}\right) Y_{q q}+\left(\frac{2}{q} G^{\prime \prime}-\frac{6}{q^{2}} G^{\prime}\right) Y_{q}\right. \\
& \left.+\left(\frac{1}{q} G^{\prime \prime \prime}-\frac{6}{q^{2}} G^{\prime \prime}+\frac{6}{q^{3}} G^{\prime}\right) Y\left(q, t_{P}\right)\right] \\
& +2 \alpha_{o}^{2}\left[\left(E^{\prime} Y_{q q q}+\left(2 E^{\prime \prime}-\frac{5}{q} E^{\prime}\right) Y_{q q}\right.\right. \\
& \left.+\left(E^{\prime \prime \prime}-\frac{5}{q^{2}} E^{\prime \prime}+\frac{5}{q^{2}} E^{\prime}\right) Y_{q}\right] \\
& +2 \beta_{o}^{2}\left[\left(\frac{5}{q} E^{\prime}\right) Y_{q q}+\left(\frac{11}{q} E^{\prime \prime}-\frac{6}{q^{2}} E^{\prime \prime}+\frac{6}{q^{3}} E^{\prime}\right) Y\left(q, t_{P}\right)\right] \\
& +Y_{q} \\
& +(17)
\end{aligned}
$$

$$
\begin{aligned}
- & 24 \beta_{o}^{2}\left[\left(\frac{1}{q^{2}} G^{\prime}\right) Y_{q}+\left(\frac{1}{q^{2}} G^{\prime \prime}-\frac{1}{q^{3}} G^{\prime}\right)\right. \\
\times & \left.Y\left(q, t_{P}\right)\right] \\
+ & 2 \alpha_{o}^{2}\left[\left(\frac{1}{q} E^{\prime}\right) Y_{q q}+\left(\frac{1}{q} E^{\prime \prime}-\frac{1}{q^{2}} E^{\prime}\right) Y_{q}\right] \\
- & 2 \beta_{o}^{2}\left[\left(\frac{5}{q^{2}} E^{\prime}\right) Y_{q}+\left(\frac{6}{q^{2}} E^{\prime \prime}-\frac{6}{q^{3}} E^{\prime}\right)\right. \\
& \left.\times\left(q, t_{P}\right)\right], \\
& \left.+6\left(\frac{1}{q} E^{\prime \prime \prime}-\frac{6}{q^{2}} E^{\prime \prime}+\frac{6}{q^{3}} E^{\prime}\right) Y\left(q, t_{S}\right)\right] \\
& +2 \beta_{o}^{2}\left[G Y_{q q q q}+\left(3 G^{\prime}-\frac{8}{q} G\right) Y_{q q q}\right. \\
& +\beta_{o}^{2}\left[E^{\prime \prime \prime}-\frac{18}{q} E_{q q q}^{\prime \prime}+\frac{46}{q^{2}} E^{\prime}\right) Y_{q} \\
& +\left(3 G^{\prime \prime}-\frac{22}{q} G^{\prime}+\frac{24}{q^{2}} G\right) Y_{q q} \\
& \left.+6\left(\frac{1}{q} G^{\prime \prime \prime}-\frac{6}{q^{2}} G^{\prime \prime}+\frac{6}{q^{3}} G^{\prime}\right) Y\left(q, t_{S}\right)\right] \\
& +\left(G^{\prime \prime \prime}-\frac{20}{q} G^{\prime \prime}+\frac{60}{q^{2}} G^{\prime}-\frac{24}{q^{3}} G\right) Y_{q} \\
& \left.+\frac{1}{q} E^{\prime \prime}-\frac{5}{q^{2}} E^{\prime}\right) Y_{q} \\
& +Y_{q q} \\
& +(19)
\end{aligned}
$$

$$
\begin{array}{rlrl}
W_{P}= & 4 \alpha_{o}^{2}\left[\left(\frac{1}{q} F\right) Y_{q q q}+\left(\frac{2}{q} F^{\prime}-\frac{4}{q^{2}} F\right) Y_{q q}\right. & W_{S}= & -2 \beta_{o}^{2}\left[G Y_{q q q q}+\left(3 G^{\prime}-\frac{6}{q} G\right) Y_{q q q}\right. \\
& \left.+\left(\frac{1}{q} F^{\prime \prime}-\frac{4}{q^{2}} F^{\prime}+\frac{4}{q^{3}} F\right) Y_{q}\right] & +\left(3 G^{\prime \prime}-\frac{18}{q} G^{\prime}+\frac{16}{q^{2}} G\right) Y_{q q}
\end{array}
$$




$$
\begin{aligned}
& +\left(G^{\prime \prime \prime}-\frac{18}{q} G^{\prime \prime}+\frac{40}{q^{2}} G^{\prime}-\frac{16}{q^{3}} G\right) Y_{q} \\
& \left.-6\left(\frac{1}{q} G^{\prime \prime \prime}-\frac{4}{q^{2}} G^{\prime \prime}+\frac{4}{q^{3}} G^{\prime}\right) Y\left(q, t_{S}\right)\right] \\
& -2 \alpha_{o}^{2}\left[\frac{1}{q} E^{\prime} Y_{q q}+\left(\frac{1}{q} E^{\prime \prime}-\frac{3}{q^{2}} E^{\prime}\right) Y_{q}\right] \\
& -\beta_{o}^{2}\left[E^{\prime} Y_{q q q}+\left(2 E^{\prime \prime}-\frac{10}{q} E^{\prime}\right) Y_{q q}\right. \\
& +\left(E^{\prime \prime \prime}-\frac{16}{q} E^{\prime \prime}+\frac{30}{q^{2}} E^{\prime}\right) Y_{q} \\
& \left.-6\left(\frac{1}{q} E^{\prime \prime \prime}-\frac{4}{q^{2}} E^{\prime \prime}+\frac{4}{q^{3}} E^{\prime}\right) Y\left(q, t_{S}\right)\right]
\end{aligned}
$$

Subscripts ' $q$ ' to $Y$ denote the derivatives of $Y(q, t)$ with respect to $q$, evaluated at $t=t_{j}$, where, $Y(q, t)=t\left[H\left(t-\left(q / \alpha_{o}\right)\right)-H\left(t-\left(q / \beta_{o}\right)\right)\right]$ and $t_{j}=t-\left(|\mathbf{r}-\mathbf{q}| / v_{j}\right),(j=P, S) . H$ is Heaviside unit step function. Primes to $F, G$ and $E$ denote their derivatives with respect to $q$.

\section{Spherical shell inhomogeneity}

To solve the equation (16), the inhomogeneity is assumed to be a spherical shell between two spheres of radii $\xi$ and $\xi+\Delta \xi$, having the same centre at the source $S$. Thus, $d V=q^{2} d q d \Omega$ with $q$ varying between the two radii. $d \Omega$ spreads over the solid angle subtended by the spherical inhomogeneity at the source. Properties of $\delta$ function help to reduce the volume integral, in equation (16), to an integral over solid angle $\Omega$. The scattering acceleration is, then, given by

$$
\begin{aligned}
\delta \ddot{u}_{i}(\mathbf{r}, t)= & u_{o} s_{o}^{2} \alpha_{o}\left[\int \chi_{f} \frac{q_{i}}{q}\left(T_{1}^{(P)}+T_{1}^{(S)}\right) d \Omega\right. \\
& \left.+n_{i} \int\left(T_{2}^{(P)}+T_{2}^{(S)}\right) d \Omega\right],
\end{aligned}
$$

where, if we define

$$
\begin{aligned}
& v_{1}(q)=\left|\frac{1}{\alpha_{o}}+X(q) \frac{\left(q-r \chi_{r}\right)}{v_{s}}\right|^{-1} ; \quad \chi_{r}=\hat{\mathbf{q}} \cdot \hat{\mathbf{r}} \\
& v_{2}(q)=\left|\frac{1}{\beta_{o}}+X(q) \frac{\left(q-r \chi_{r}\right)}{v_{s}}\right|^{-1}
\end{aligned}
$$

$$
\begin{aligned}
q_{o}= & {\left[r \chi_{r}-t v_{s} v_{r}+\left\{r^{2}\left(\chi_{r}^{2}+v_{r}^{2}-1\right)+v_{s}^{2} t^{2}\right.\right.} \\
& \left.\left.-2 r \chi_{r} v_{s} t v_{r}\right\}^{1 / 2}\right] /\left(1-v_{r}^{2}\right) \\
q_{o o}= & 0.5\left(r^{2}-t^{2} v_{s}^{2}\right) /\left(r \chi_{r}-t v_{s}\right)
\end{aligned}
$$

then

$$
\begin{aligned}
T_{1}^{(P)}= & v_{2}\left(q_{2}\right) V_{11}\left(q_{2}\right) \frac{q_{2}}{\beta_{o}^{2}}-v_{1}\left(q_{1}\right) V_{11}\left(q_{1}\right) \frac{q_{1}}{\alpha_{o}^{2}} \\
& +\int_{q_{1}}^{q_{2}}\left(t-\frac{|\mathbf{r}-\mathbf{q}|}{\alpha_{o}}\right) V_{12}(q) d q, \\
T_{2}^{(P)}= & v_{2}\left(q_{2}\right) W_{11}\left(q_{2}\right) \frac{q_{2}}{\beta_{o}^{2}}-v_{1}\left(q_{1}\right) W_{11}\left(q_{1}\right) \frac{q_{1}}{\alpha_{o}^{2}} \\
& +\int_{q_{1}}^{q_{2}}\left(t-\frac{|\mathbf{r}-\mathbf{q}|}{\alpha_{o}}\right) W_{12}(q) d q,
\end{aligned}
$$

with $v_{s}=\alpha_{o}, v_{r}=\alpha_{o} / \beta_{o}, q_{1}=q_{o o}$ and $q_{2}=q_{o}$; and

$$
\begin{aligned}
T_{1}^{(S)}= & v_{2}\left(q_{2}\right) V_{21}\left(q_{2}\right) \frac{q_{2}}{\beta_{o}^{2}}-v_{1}\left(q_{1}\right) V_{21}\left(q_{1}\right) \frac{q_{1}}{\alpha_{o}^{2}} \\
& +\int_{q_{1}}^{q_{2}}\left(t-\frac{|\mathbf{r}-\mathbf{q}|}{\beta_{o}}\right) V_{22}(q) d q, \\
T_{2}^{(S)}= & v_{2}\left(q_{2}\right) W_{21}\left(q_{2}\right) \frac{q_{2}}{\beta_{o}^{2}}-v_{1}\left(q_{1}\right) W_{21}\left(q_{1}\right) \frac{q_{1}}{\alpha_{o}^{2}} \\
& +\int_{q_{1}}^{q_{2}}\left(t-\frac{|\mathbf{r}-\mathbf{q}|}{\beta_{o}}\right) W_{22}(q) d q,
\end{aligned}
$$

with $v_{s}=\beta_{o}, v_{r}=\beta_{o} / \alpha_{o}, q_{1}=q_{o}$ and $q_{2}=q_{o o}$.

Other functions, used in (26)-(29), are given by

$$
\begin{aligned}
V_{11}(q)= & -4 \alpha_{o}^{2}\left(\frac{1}{q} X_{3}+\frac{5}{q^{2}} X_{2}\right. \\
& \left.-\frac{2}{q^{3}} X_{1}-\frac{6}{q^{4}} X\right) F(q) \\
& +2 \alpha_{o}^{2}\left(\frac{1}{q} X_{2}+\frac{3}{q^{2}} X_{1}-\frac{3}{q^{3}} X\right) E^{\prime}
\end{aligned}
$$


406

$$
\begin{aligned}
& -\frac{24}{q^{2}} \beta_{o}^{2}\left[X G^{\prime \prime}-\left(X_{1}+\frac{4}{q} X\right) G^{\prime}\right] \\
& -\frac{2}{q^{2}} \beta_{o}^{2}\left[6 X E^{\prime \prime}-\left(5 X_{1}+\frac{21}{q} X\right) E^{\prime}\right], \\
& V_{12}(q)=-\beta_{o}^{2}\left(\frac{12}{q^{2}}\right)\left[2\left(G^{\prime \prime \prime}-\frac{6}{q} G^{\prime \prime}+\frac{6}{q^{2}} G^{\prime}\right)\right. \\
& \left.+\left(E^{\prime \prime \prime}-\frac{6}{q} E^{\prime \prime}+\frac{6}{q^{2}} E^{\prime}\right)\right] X, \\
& W_{11}(q)=\alpha_{o}^{2} \frac{2}{q^{2}}\left[2\left(X_{2}-\frac{2}{q^{2}} X\right) F(q)\right. \\
& \left.-\left(X_{1}-\frac{1}{q} X\right) E^{\prime}\right] \\
& -\beta_{o}^{2} \frac{2}{q^{3}}\left[12 G^{\prime}+5 E^{\prime}\right] X \\
& W_{12}(q)=-\beta_{o}^{2}\left(\frac{12}{q^{3}}\right)\left[2\left(G^{\prime \prime}-\frac{1}{q} G^{\prime}\right)\right. \\
& \left.+\left(E^{\prime \prime}-\frac{1}{q} E^{\prime}\right)\right] X \\
& V_{21}(q)=-2 \beta_{o}^{2}\left[\frac{6}{q^{2}} X G^{\prime \prime}-\frac{6}{q^{2}}\left(X_{1}+\frac{4}{q} X\right) G^{\prime}\right. \\
& \left.+\left(\frac{1}{q} X_{3}+\frac{5}{q^{2}} X_{2}-\frac{2}{q^{3}} X_{1}-\frac{6}{q^{4}} X\right) G(q)\right] \\
& -2 \alpha_{o}^{2}\left(\frac{1}{q^{2}}\left(X_{1}+\frac{3}{q} X\right) E^{\prime}-\beta_{o}^{2}\left[\frac{6}{q^{2}} X E^{\prime \prime}\right.\right. \\
& \left.-\left(\frac{1}{q} X_{2}+\frac{10}{q^{2}} X_{1}+\frac{24}{q^{3}} X\right) E^{\prime}\right], \\
& V_{22}(q)=-\beta_{o}^{2}\left(\frac{6}{q^{2}}\right)\left[2\left(G^{\prime \prime \prime}-\frac{6}{q} G^{\prime \prime}+\frac{6}{q^{2}} G^{\prime}\right)\right. \\
& \left.+\left(E^{\prime \prime \prime}-\frac{6}{q} E^{\prime \prime}+\frac{6}{q^{2}} E^{\prime}\right)\right] X,
\end{aligned}
$$

\section{D Sharma}

$$
\begin{aligned}
W_{21}(q)= & 2 \beta_{o}^{2}\left[\frac{6}{q^{2}} X G^{\prime \prime}-\frac{6}{q^{2}}\left(X_{1}+\frac{2}{q} X\right) G^{\prime}\right. \\
& \left.+\left(\frac{1}{q} X_{3}+\frac{3}{q^{2}} X_{2}-\frac{2}{q^{3}} X_{1}-\frac{2}{q^{4}} X\right) G(q)\right] \\
& +\alpha_{o}^{2} \frac{2}{q^{2}}\left(X_{1}+\frac{1}{q} X\right) E^{\prime}+\beta_{o}^{2}\left[\frac{6}{q^{2}} X E^{\prime \prime}\right. \\
& \left.-\left(\frac{1}{q} X_{2}+\frac{8}{q^{2}} X_{1}+\frac{12}{q^{3}} X\right) E^{\prime}\right], \\
W_{22}(q)= & \beta_{o}^{2}\left(\frac{6}{q^{2}}\right)\left[2\left(G^{\prime \prime \prime}-\frac{4}{q} G^{\prime \prime}+\frac{4}{q^{2}} G^{\prime}\right)\right. \\
& \left.+\left(E^{\prime \prime \prime}-\frac{4}{q} E^{\prime \prime}+\frac{4}{q^{2}} E^{\prime}\right)\right] X,
\end{aligned}
$$

where

$$
\begin{aligned}
& X_{1}=-\left(q-r \chi_{r}\right) X^{3} \\
& X_{2}=-X^{3}+3\left(q-r \chi_{r}\right)^{2} X^{5}, \\
& X_{3}=9\left(q-r \chi_{r}\right) X^{5}-15\left(q-r \chi_{r}\right)^{3} X^{7} .
\end{aligned}
$$

\section{Special cases}

- Smooth variations of density inhomogeneity (i.e., $\left.E^{\prime}(q)=0\right)$ reduces the expressions of $V_{i j}, W_{i j}$, $(i, j=1,2)$, to a great extent.

- The back scattering expression for acceleration is expressed as follows:

$$
\begin{aligned}
\delta \ddot{u}_{i}(\mathbf{r}, t)= & u_{o} s_{o}^{2} \alpha_{o}\left[\left(T_{1}^{(P)}+T_{1}^{(S)}\right) \int \chi_{f} \frac{q_{i}}{q} d \Omega\right. \\
& \left.+n_{i}\left(T_{2}^{(P)}+T_{2}^{(S)}\right) \int d \Omega\right],
\end{aligned}
$$

where $q_{o}=t v_{s} /\left(1+v_{r}\right), q_{o o}=0.5 t v_{s}$, and in the case of smooth density inhomogeneity,

$$
\begin{aligned}
V_{11}(q) & =-\beta_{o}^{2} \frac{24}{q^{3}}\left(G^{\prime \prime}-\frac{3}{q} G^{\prime}\right) \\
V_{12}(q) & =-\beta_{o}^{2} \frac{24}{q^{3}}\left(G^{\prime \prime \prime}-\frac{6}{q} G^{\prime \prime}+\frac{6}{q^{2}} G^{\prime}\right) ; \\
W_{11}(q) & =-\beta_{o}^{2} \frac{24}{q^{4}} G^{\prime}
\end{aligned}
$$



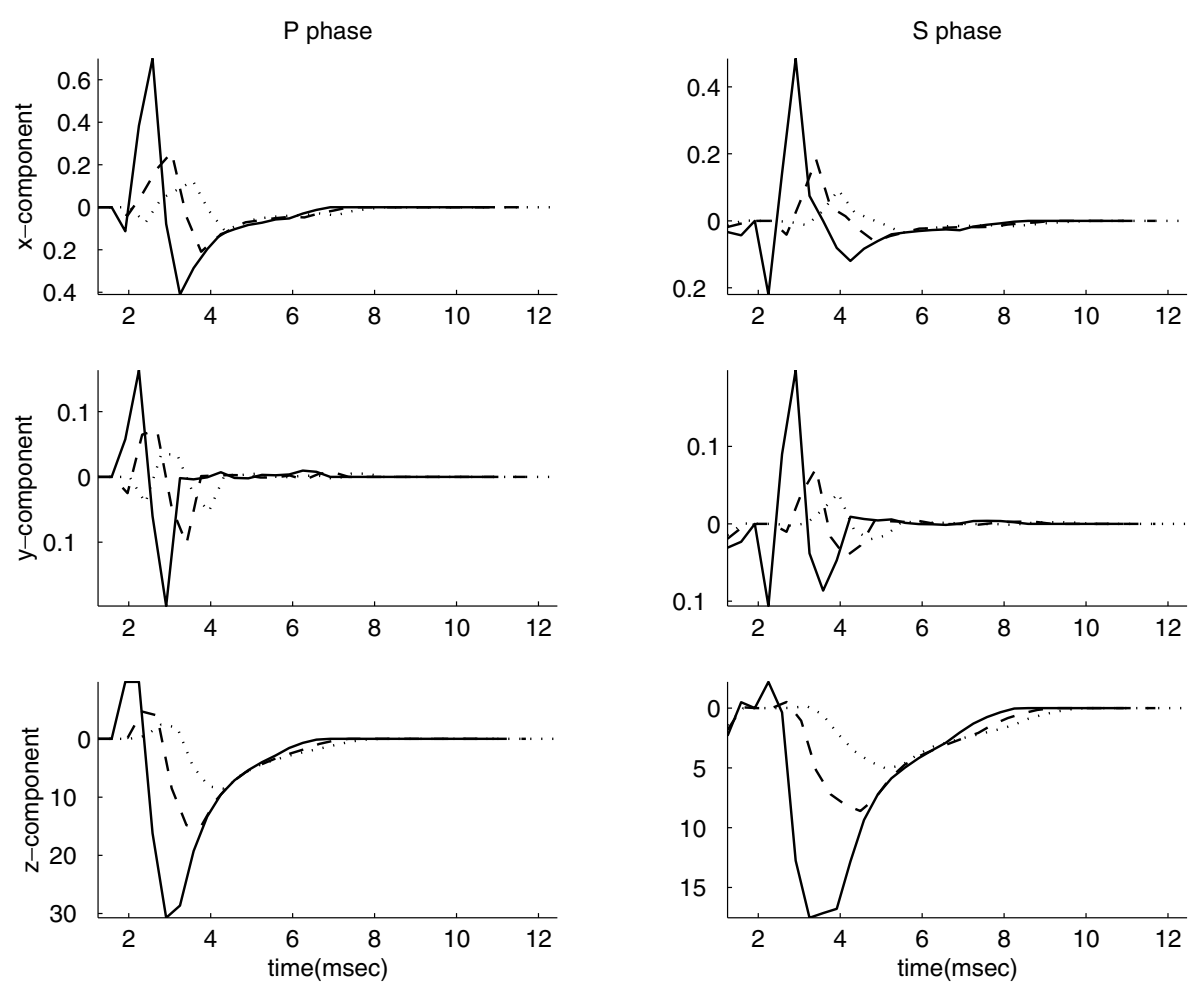

Figure 2. Variations in scattering acceleration with the source-inhomogeneity distance $\{\xi=4 \mathrm{~m}:-; \quad \xi=5 \mathrm{~m}:-----;$ $\xi=6 \mathrm{~m}:$........... $\}$.
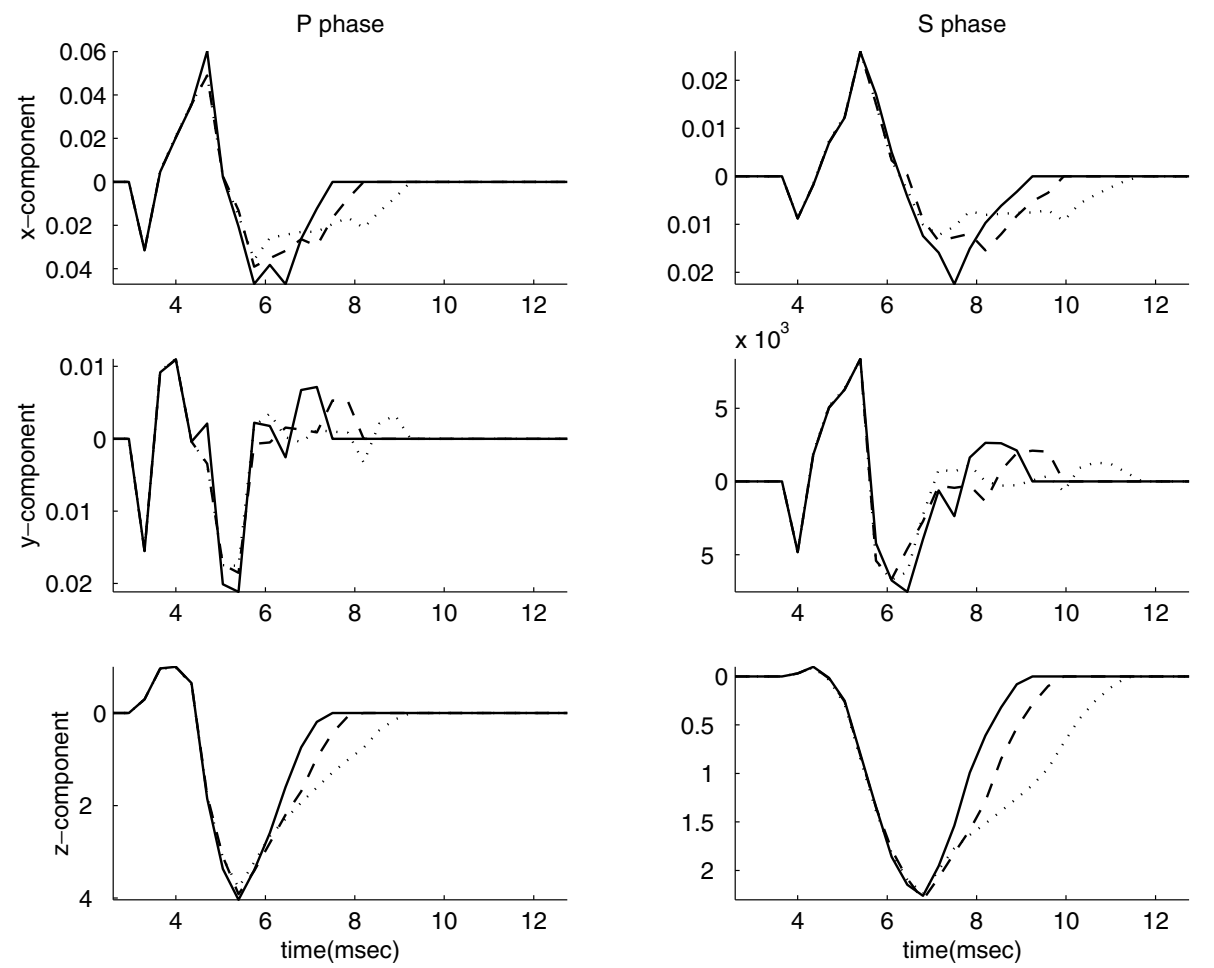

Figure 3. Variations in scattering acceleration with radial thickness of inhomogeneity $\left\{\Delta \xi=3 \mathrm{~m}:-\frac{-}{-} \Delta \xi=4 \mathrm{~m}:-----\right.$; $\Delta \xi=6 \mathrm{~m}$ : ... . 

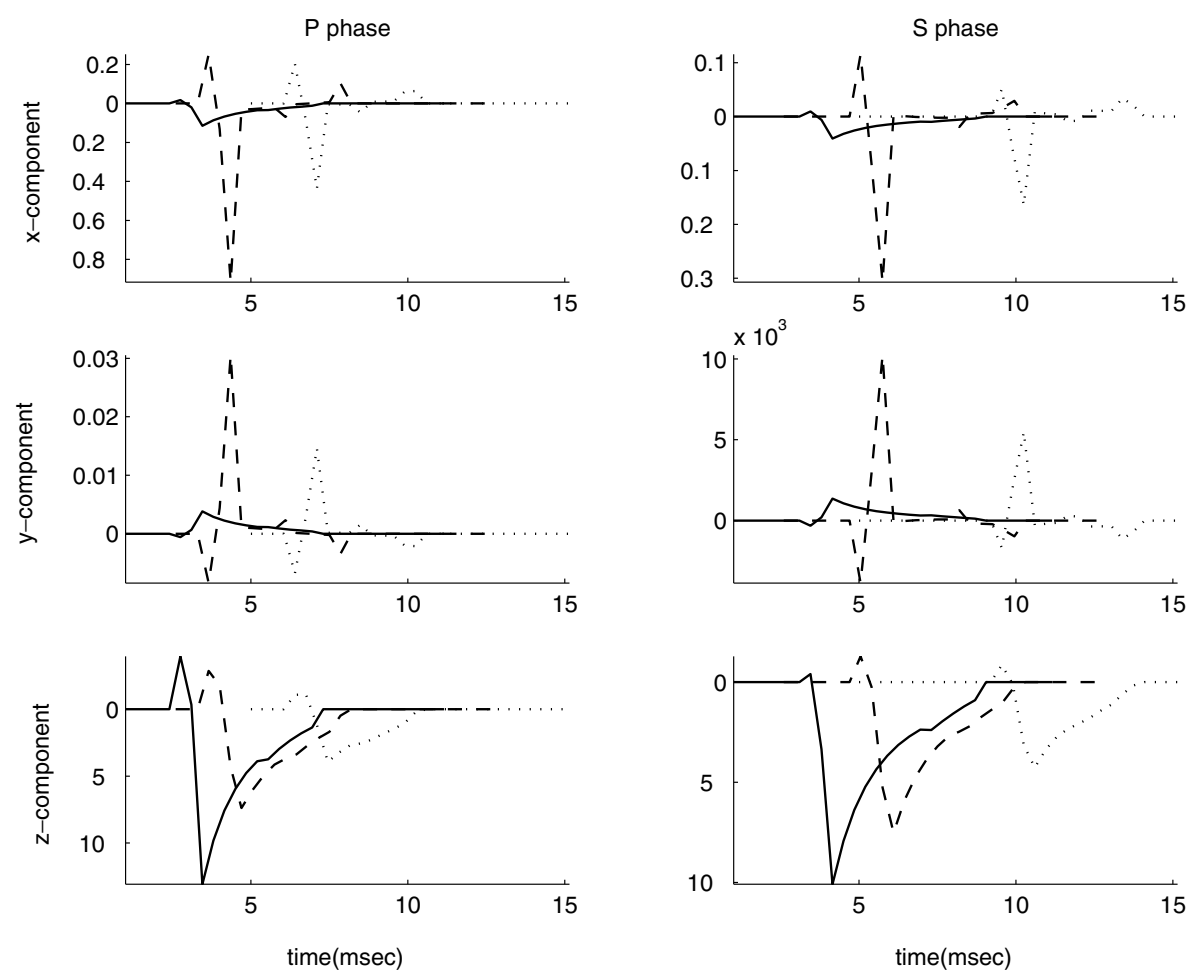

Figure 4. Variations in scattering acceleration with the source-receiver distance $\{r=0.001 \mathrm{~m}:-; \quad r=8 \mathrm{~m}:-----$; $r=20 \mathrm{~m}:$........... $\}$.
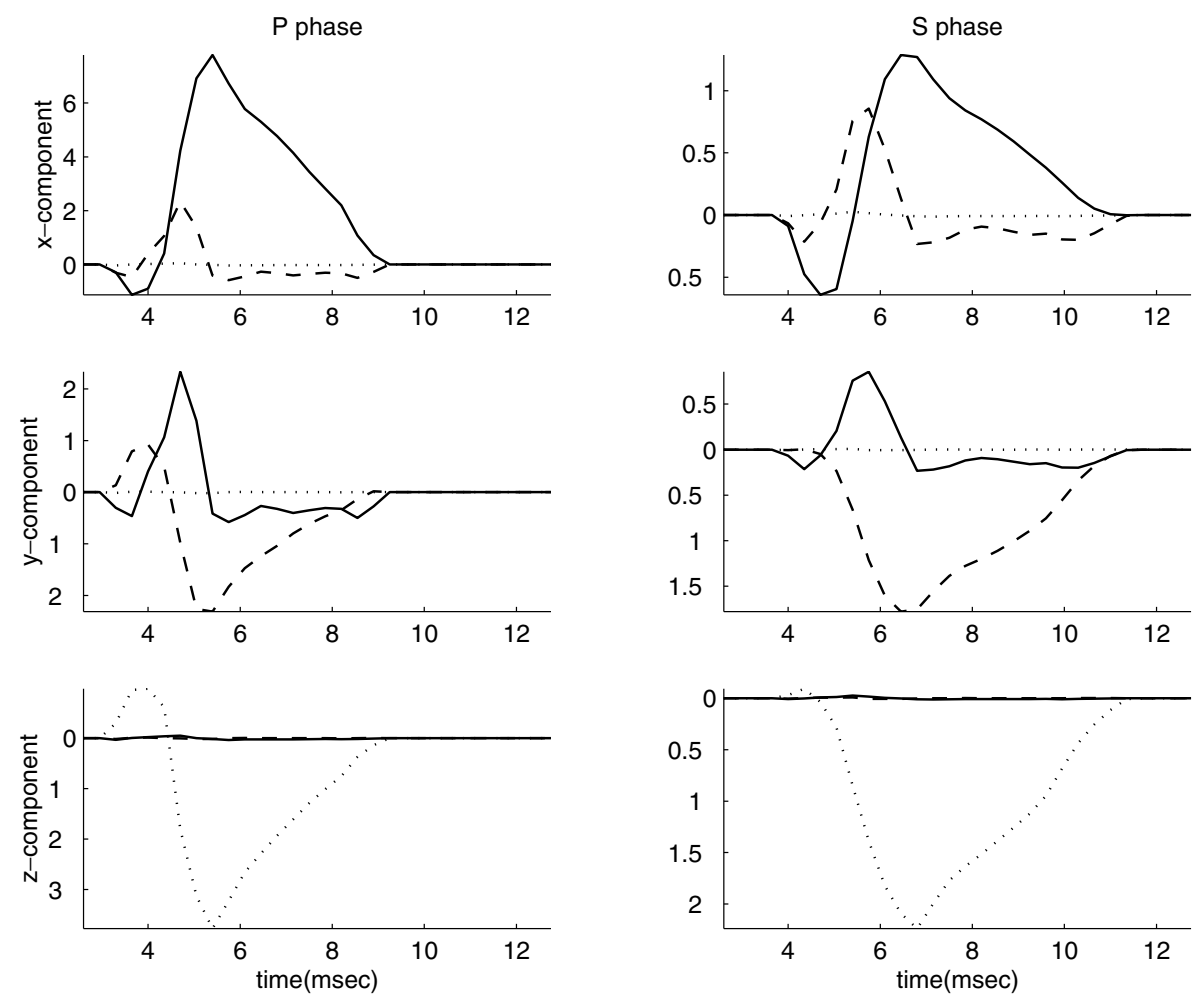

Figure 5. Variations in scattering acceleration with the direction of force $\{n=(1,0,0):-; \quad n=(0,1,0):----$; $n=(0,0,1): \ldots \ldots \ldots . .$.$\} .$ 


$$
\begin{aligned}
& W_{12}(q)=-\beta_{o}^{2} \frac{24}{q^{4}}\left(G^{\prime \prime}-\frac{1}{q} G^{\prime}\right) ; \\
& V_{21}(q)=0.5 V_{11}(q) ; \quad V_{22}(q)=0.5 V_{12}(q) ; \\
& W_{21}(q)=\beta_{o}^{2} \frac{12}{q^{3}}\left(G^{\prime \prime}-\frac{1}{q} G^{\prime}\right) ; \\
& W_{22}(q)=\beta_{o}^{2} \frac{12}{q^{3}}\left(G^{\prime \prime \prime}-\frac{4}{q} G^{\prime \prime}+\frac{4}{q^{2}} G^{\prime}\right) .
\end{aligned}
$$

It may be noted that the above expressions are independent of function $F(q)$ and its derivatives. This implies that acceleration for first order scattering from a spherical shell inhomogeneity is not affected by the $P$-wave velocity inhomogeneity. The effect of $S$ wave velocity inhomogeneity is represented by the derivatives of $G(q)$ which means the inhomogeneity with smooth perturbation of shear wave velocity will not back-scatter the nearfield of a point source. Hence, the accelerations of back-scattered near-field are controlled only by the derivatives of $G(q)$. Similar results are obtained by Leary (1995) for the scattering of far-field shear motion. Even, for a radial inhomogeneity of any shape and size, the scattered $S$ acceleration (i.e., equations (19) and (20)) is independent of $P$ wave velocity inhomogeneity. Moreover, the integrals in (30) are simple enough to be solved analytically over any given size of spherical shell inhomogeneity.

\section{Numerical results and discussion}

The purpose of numerical computation is to explore the use of expressions derived in the simulation of the records of near-fault observations. The numerical study of the effects of various parameters on scattered acceleration, also, verifies the correctness of the expressions derived. This requires the integral expressions (21)-(29) to be solved over the volume of the inhomogeneity. In a hypothetical model, the spherical co-ordinate system is chosen with its origin at the source. The inhomogeneity is only a part of spherical shell defined by $\chi$ varying from -0.1 to 0.1 and $\phi$ varying from $-0.2 \pi$ to $0.2 \pi$. Density perturbation is assumed constant to simplify the computation. The perturbations in wave velocities are defined by $F(q)=G(q)=0.05 \sin k(q-\xi)$. This implies that the velocity perturbation is zero at the inner surface of the inhomogeneity and changes outward with sine function. Wave velocities in the homogeneous surrounding are $\alpha_{o}=4 \mathrm{~km} / \mathrm{sec}$, and $\beta_{o}=$ $2.5 \mathrm{~km} / \mathrm{sec}$. Numerical integrations over $d q$ and $d \Omega(=d \chi d \phi)$, are computed through summation method.

To study the effects of size $(\Delta \xi)$, distance $(\xi)$ and position (r) of inhomogeneity from source and the direction of force $(\hat{\mathbf{n}})$ on scattering, the computed phases are exhibited in figures $2-5$. Details are as follows.

All the three components of acceleration are plotted, phase-wise, in figure 2, for values of $\xi=4,5, \quad 6 \mathrm{~m} . k=0.1, \Delta \xi=6 \mathrm{~m}, \quad r=5 \mathrm{~m}$, $\hat{\mathbf{r}}=(1,1,1) / \sqrt{3}$ and $\hat{\mathbf{n}}=(0,0,1)$. The scattering decreases rapidly with the increase of $\xi$. The direction of force being along $z$-direction, $x$ - and $y$-components of acceleration are negligible as compared to its $z$-component. Peak acceleration for $P$ phase is nearly twice that for $S$ phase.

The effect of thickness of inhomogeneity on scattered acceleration is presented in figure 3 , for the values of $\Delta \xi=3,4,6 \mathrm{~m} . \xi=8 \mathrm{~m}$ and $r=5 \mathrm{~m}$. As expected the initial part of the signal is common and the effect of increase of $\Delta \xi$ is observed, only in the later part. Alongwith the increase of scattering, the increase of $\Delta \xi$ also increases the signal duration.

Figure 4 contains the variations of accelerations with the distance of receiver from source. For an easy comparison with the back scattering the receiver location is restricted to the negative direction of polar axis. Hence, $\hat{\mathbf{r}}=(0,0,-1)$. $\xi=5 \mathrm{~m}, \Delta \xi=6 \mathrm{~m}$ and computations are made for values of $r=0.001,8,20 \mathrm{~m}$. In the present geometry, $x$ - and $y$-components of scattered acceleration may increase but resultant of scattered acceleration decreases with the increase of $r$.

To study the effect of position on inhomogeneity with respect to force orientation, $\hat{\mathbf{n}}$ is taken along different axes. Accelerations are plotted in figure 5 . The value of parameters are not changed except $\xi=8 \mathrm{~m}$, and $r=5 \mathrm{~m}$. Scattered $S$ phase components are smaller as compared to $P$ phase components. When the force is along $z$-direction, negligible motion is observed in $x-y$ plane. Conversely, when the force is in $x-y$ plane, there may not be any considerable acceleration along $z$-direction.

\section{Conclusions}

The analytical expressions derived in previous sections are interpreted to characterise the behaviour of the first order scattering of near-field. It is noted that, in general, the behaviour of $P$ and $S$ phases of scattered acceleration is similar to the behaviour of dilatation and rotation, respectively, of scattered waves studied in Sharma (2004). For example, the acceleration of $S$ waves scattered from a radial inhomogeneity is independent of $P$ wave velocity 
inhomogeneity and the acceleration of scattered $P$ motion does not depend upon $S$ wave velocity inhomogeneity but its gradient. The expressions for back-scattering may be interpreted for the following conclusions:

- Acceleration of back scattering from a radially inhomogeneous spherical shell is independent of $P$ wave velocity inhomogeneity.

- A radial inhomogeneity with smooth variations of $S$ wave velocity does not back-scatter the near-field of a point source.

The numerical results are obtained for a very particular hypothetical model. The quantitative interpretation of these results should not be generalised. However, qualitative behaviour of these results may be of some use in simulation studies. Here, also, the effects of source-receiver distance and source-scatterer distance on scattered acceleration are similar to their effects on scattered strains (Sharma 2004). The few additional observations made out of the discussion of numerical results are as follows.

- The increase in the thickness of spherical shell may increase the scattered motion. It, also, increases the duration of the signal.

- The scattering acceleration is confined to the $x-y$ plane when the force is along $x$ - or $y$-direction. However, for scattering due to $z$-component of force, the acceleration is parallel to the force.

The work presented studies the scattering of near-field of a point source in time domain. The expressions derived may be used to calculate the acceleration of scattered phases without any restriction on the locations of source and receiver. Back scattering expressions are obtained in simple closed form. Acceleration, being a directly measurable quantity, can be used to investigate the physical phenomenon that shapes the relations between material response and source properties. A major application of scattering acceleration may be the ultrasonic detection and characterisation of flaws in elastic materials (Rose 1989). Near-field accelerograms may be used, more effectively, to simulate and interpret the time-lapse borehole data from a uniwell or crosswell survey (Paulsson et al 1994) for reservoir characterisation. The earthquake/structural engineers may be interested in the realistic ground accelerations that are site specific. A reliable interpretation of the ground motions can be obtained by the use of arrays of strong motion accelerometers (Iwan 1978). Hence, this study may, also, be useful in the modeling of accelerograms to predict the seismic hazards due to an impending earthquake in any region.

\section{References}

Aki K and Richards P G 1980 Quantitative Seismology, Theory and Methods, Vol. I \& II (New York: Freeman).

Iwan W D (ed.) 1978 Strong-Motion Earthquake Instrum. Arrays: Proc. Int. Workshop Strong-Motion Earthquake Instrum. Arrays (Hawaii: Honolulu).

Leary P C 1995 Quantifying crustal fracture heterogeneity by seismic scattering; Geophys. J. Int. 122 125-142.

Paulsson B N P, Meredith J A, Wang Z and Fairborn J W 1994 The Steepbank crosswell seismic project: Reservoir definition and evaluation of steamflood technology in Alberta tar sands; The Leading Edge 13 737-747.

Rose J H 1989 Elastic wave inverse scattering in nondestructive evaluation; PAGEOPH 131 715-739.

Sato H 1984 Attenuation and envelope formation of three component seismograms of small local earthquakes in randomly inhomogeneous lithosphere; J. Geophys. Res. 89 1221-1242.

Sharma M D 2004 Strains of scattering of a near-field of a point source; Proc. Indian Acad. Sci. (Earth Planet. Sci.) 113(2) 247-257.

Wu R S 1989 The perturbation method in elastic wave scattering; PAGEOPH 131 605-637.

Wu R S and Aki K 1985a Scattering characteristics of elastic waves by an elastic heterogeneity; Geophysics $\mathbf{5 0}$ $582-595$.

Wu R S and Aki K 1985b Elastic wave scattering by a random medium and the small scale inhomogeneities in the lithosphere; J. Geophys. Res. 90 10261-10273.

Wu R S and Aki K 1988 Introduction: Seismic wave scattering in three-dimensionally heterogeneous earth; $P A G E O P H 128$ 1-6. 